\title{
Diseño y control de un convertidor de energía eléctrica a térmica, usando como fuente un sistema híbrido eólico-solar
}

\author{
Design and control of an electric to thermal energy converter, using a \\ wind-solar hybrid system as source
}
Carlos Mauricio Carrillo Rosero. ${ }^{1}$, Alexis Abraham Aldaz Toaza. ${ }^{2}$, Luis David Moreta López. $^{3} \&$ Alex Santiago Mayorga Pardo. ${ }^{4}$

Recibido: 11-02-2021 / Revisado: 20-02-2021 /Aceptado: 12-03-2021/ Publicado: 05-04-2021

\begin{abstract}
. DOI: https://doi.org/10.33262/concienciadigital.v4i2.1657

Introduction: This research is focused on satisfying the basic energy needs of a small house that did not have electricity, located in the Río Blanco community of Yatzaputzán, Tungurahua province. Initially, and due to economic, geographical and environmental conditions, as a cold moor-type climate prevails, a hybrid wind-photovoltaic system was designed and installed for battery charging, which made it possible to provide lighting and use of electrical appliances; as well as providing hot water through a preheating system consisting of vacuum tubes and flat plate thermal panels. Objective: To generate electrical energy that allows adequate battery charging by applying a control system, in order to optimize operating periods of a converter that uses electrical resistors and converts them into thermal energy. Methodology: The tests were carried out by taking data in situ, considering the parameters of solar radiation, wind speed and average ambient temperature. Results: From the analysis carried out, it was obtained that the

\footnotetext{
1 Universidad Técnica de Ambato, Facultad de Ingeniería Civil y Mecánica, Carrera de Ingeniería Mecánica. Ambato, Ecuador. cmcarrillo@uta.edu.ec, http://orcid.org/0000-0001-6578/9362

2 Universidad Técnica de Ambato, Facultad de Ingeniería Civil y Mecánica, Carrera de Ingeniería Mecánica. Ambato, Ecuador. aaldaz5580@uta.edu.ec, https://orcid.org/0000-0002-3992-0803

3 Universidad Técnica de Ambato, Facultad de Ingeniería Civil y Mecánica, Carrera de Ingeniería Mecánica. Ambato, Ecuador. luisdmoretal@gmail.com, https://orcid.org/0000-0001-5173-0765

4 Universidad Técnica de Ambato, Facultad de Ingeniería Civil y Mecánica, Carrera de Ingeniería Mecánica. Ambato, Ecuador. asmayorga@uta.edu.ec, https://orcid.org/0000-0001-8875-8470
} 
monthly average of the wind speed is 1.5 to $2 \mathrm{~m} / \mathrm{s}$ with a reference height of $2.50 \mathrm{~m}$ and the average monthly radiation takes values between 140 to $150 \mathrm{~W} / \mathrm{m}^{2}$. To guarantee the correct operation of the converter, projections were made to determine the increase in water temperature, showing that the project has a high efficiency. Conclusions: It was established that by carrying out a preheating process and a constant supply of electrical energy from the batteries and generated by unconventional means with ambient temperature and initial voltage conditions in the Río Blanco community, they produce an average increase in temperature in the operation of the converter in the range of 2 to $4^{0} \mathrm{C}$.

Keywords: Energy converter, hybrid system, photovoltaic energy, wind energy.

\section{Resumen.}

Introducción: La presente investigación está enfocada en satisfacer necesidades energéticas básicas de una pequeña vivienda que no disponía de energía eléctrica ubicada en el sector de Río Blanco comunidad de Yatzaputzán provincia de Tungurahua. Inicialmente y debido a condiciones económicas, geográficas y ambientales al prevalecer un clima frío de tipo páramo se diseñó e instaló un sistema híbrido eólico-fotovoltaico para la carga de baterías que permitió dotar de iluminación y utilización de electrodomésticos; así como también el otorgar agua caliente mediante un sistema de precalentamiento que consiste de tubos al vacío y paneles térmicos de placa plana Objetivo: Generar energía eléctrica que permita la carga adecuada de baterías aplicando un sistema de control, con la finalidad de optimizar periodos de funcionamiento de un convertidor que utiliza resistencias eléctricas y las convierte en energía térmica. Metodología: Las pruebas se realizaron mediante la toma de datos in situ, considerando los parámetros de radiación solar, velocidad del viento y temperatura ambiente promedio. Resultados: Del análisis realizado se obtuvo que el promedio mensual de la velocidad del viento es de 1.5 a $2 \mathrm{~m} / \mathrm{s}$ con una altura de referencia de $2.50 \mathrm{~m}$ y la radiación mensual promedio toma valores comprendidos entre 140 a $150 \mathrm{~W} / \mathrm{m}^{2}$. Para garantizar el correcto funcionamiento del convertidor se realizaron proyecciones que permiten determinar el incremento de temperatura del agua demostrando que la realización del proyecto tiene una eficiencia alta. Conclusiones: Se estableció que al realizar un proceso de precalentamiento y un suministro constante de energía eléctrica provenientes de las baterías y generados por medios no convencionales con condiciones de temperatura ambiente y voltaje inicial en la comunidad de Río Blanco producen en promedio un incremento de temperatura en el funcionamiento del convertidor en el rango de 2 a $4{ }^{\circ} \mathrm{C}$

Palabras claves: Convertidor de energía, sistemas híbridos, energía fotovoltaica, energía eólica.

\section{Introducción.}

Se conoce a un sistema híbrido como un sistema de energía renovable que tiene dos fuentes de generación, este tipo de sistemas de generación tiene una mayor eficacia y 
eficiencia, ya que disminuye la cantidad de baterías que se deben emplear para un sistema aislado. La radiación solar y la velocidad del viento se pueden ver complementadas debido a que durante el día sin considerar las horas solares pico y la variación del viento, en todo momento uno de estos recursos se encuentra presente y permite continuar con la generación de energía eléctrica de manera ininterrumpida, variando únicamente su intensidad. (Colmenar, Borge, Collado, \& Castro, 2015) (Prats, García, \& Alonso, 2011).

Se requiere de un potencial eléctrico de 522 Wh/día (Guananga, 2017), para satisfacer las exigencias a las que estará expuesto el sistema híbrido de generación. En la Figura 1, se esquematiza los componentes de todo el sistema híbrido, las conexiones son un punto de partida fundamental en la etapa de diseño del convertidor debido a que se puede emplear el circuito para determinar la mejor posibilidad de conexiones del sistema.

La función principal del convertidor de voltaje es la de receptar y convertir toda la energía eléctrica que ha sido captada por el sistema híbrido de generación instalado en la comunidad de Rio Blanco, los valores nominales de tensión alcanzan valores de 20,6 voltios y 4,15 amperios en condiciones climáticas favorables.
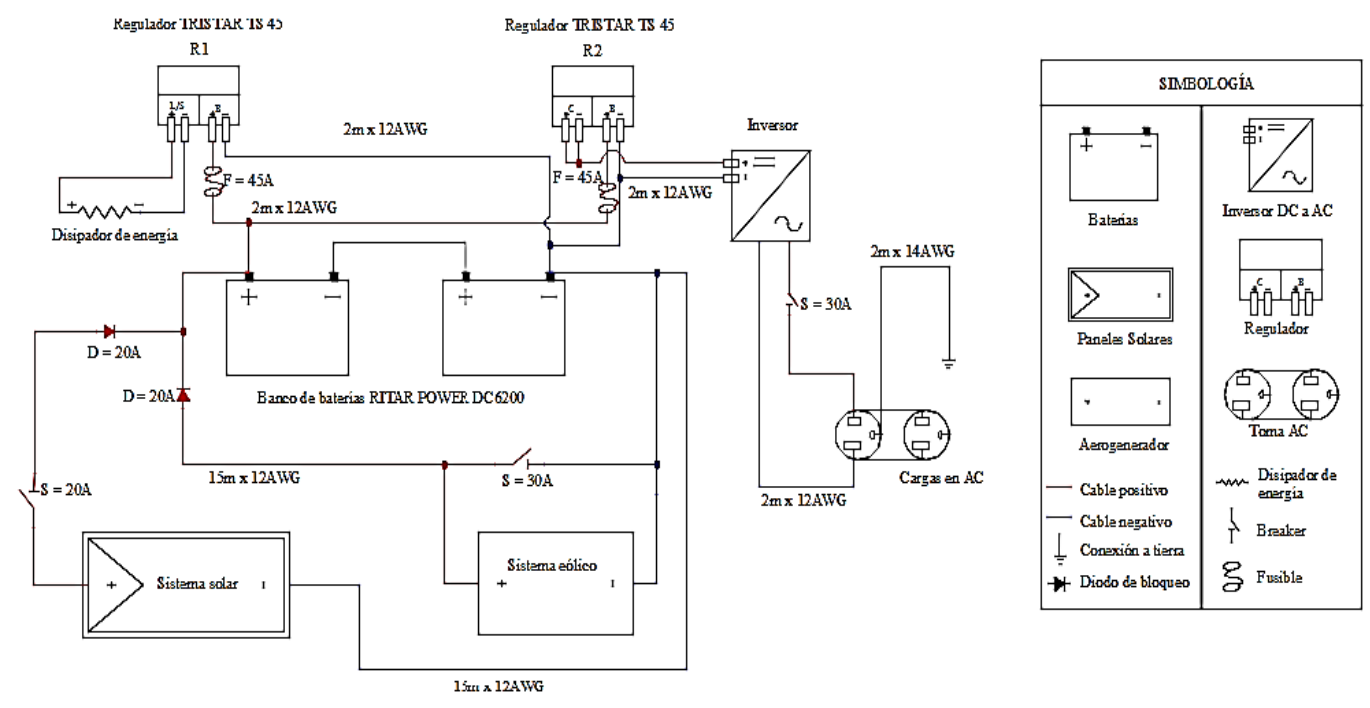

Figura 1. Circuito de conexiones del sistema híbrido

Fuente: (Guananga, 2017)

Tal como menciona (Ogata, 2010) un sistema de control de lazo cerrado es aquel que posee retroalimentación relacionando entre sí su señal de entrada con su señal de salida. En el proyecto planteado la señal de entrada queda determinada por el nivel de carga en las baterías. El sistema provisto de un controlador Arduino UNO basada en un microcontrolador Atmega 328 (Geek Factory, s.f.) al poseer entradas analógicas de 0 a 5 voltios no necesita que se acople al mismo un sensor específico para la lectura de carga ya que se la puede realizar directamente por estas entradas utilizando para ello un divisor de voltaje. Este controlador es el encargado de mantener el nivel de carga y reaccionar ante la descarga cortando la salida de energía hasta que se encuentre en el nivel programado aceptado. Y para la adquisición de datos un módulo Ethernet Shield con 
capacidad de soportar TCP y UDP provista de una pila de red IP y cuatro sockets de conexión. (Ethernet library, s.f.)

\section{Metodologia.}

Se empieza por describir el sistema eólico-solar encargado de suministrar energía eléctrica y a continuación el sistema de precalentamiento de agua. El sistema híbrido cuenta con un pequeño aerogenerador de imanes permanentes y de flujo axial que está diseñado para bajas velocidades de viento colocado a 11 metros de altura, con un rotor de tres álabes de 2,6 metros de diámetro. Con base a la curva de potencia obtenidas por (Espín, 2019) (Naranjo, 2017) indican que el generador eólico de baja potencia se encuentra en su óptimo funcionamiento alcanzando una potencia de diseño de $178 \mathrm{~W}$.

De acuerdo a (Carrillo, Romero, \& Mayorga, 2020) se determinó que la velocidad de viento a la altura del buje entregada por el generador eólico durante un tiempo de muestreo en la zona está comprendida en un rango de 2 a $12 \mathrm{~m} / \mathrm{s}$ con una velocidad de giro del rotor de 2 a $120 \mathrm{rpm}$, generando un voltaje de 0.5 a $16 \mathrm{~V}$ y desde 0.5 a $13 \mathrm{~A} \mathrm{El}$ sistema híbrido cuenta también con dos paneles fotovoltaicos de $100 \mathrm{~W}$ marca SUNLINK SL080-12M100 de alta eficiencia y durabilidad de 10 años los cuales son de fácil instalación y se pueden adaptar a diversas aplicaciones como soportar cargas producidas por viento, nieve o neblina. (Espín, 2019)

En un proceso de calentamiento de agua cuando se utiliza fuentes de 12 voltios se pueden obtener potencias de $600 \mathrm{~W}$ que están muy por debajo de potencias de $4500 \mathrm{~W}$ que se pueden alcanzar con tensiones de 110 y 220 voltios, por esta razón en el proyecto se optó por adecuar dos sistemas independientes de paneles térmicos en cuyo interior se encuentra un mecanismo de termosifón que permite que en el precalentamiento se eleve la temperatura a los grados centígrados requeridos y se mantenga dentro de un rango adecuado de temperatura de confort. Un mecanismo de termosifón tiene como principio el cambio de densidad del fluido (agua); si se eleva su temperatura, este se dilata provocando que el fluido a menor temperatura converja hacia el captador tal como se muestra en la figura 2 generalmente se realiza por convección y para que se produzca la recirculación se acopla una bomba eléctrica con la finalidad de que se dé un equilibrio térmico dentro del convertidor.

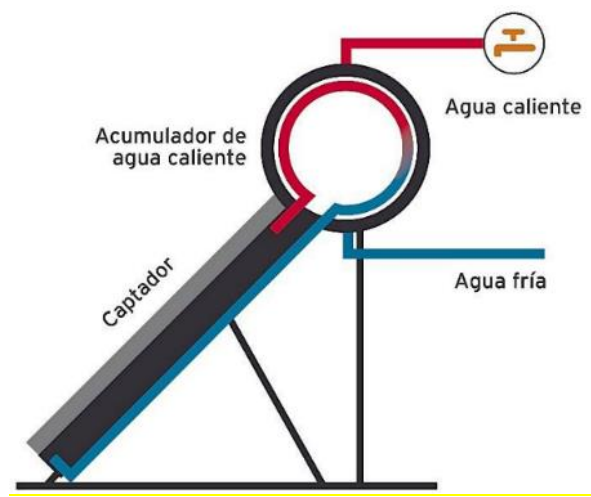

Figura 2. Esquema de termosifón

Fuente: (Méndez \& García, 2007) 
Las especificaciones de cada uno de los dos sistemas independientes son:

- El panel térmico Figura 3, es un colector solar plano con un área efectiva de $1.0 \mathrm{~m}^{2}$, si se considera al tiempo de exposición a la radiación solar como su característica principal, de las pruebas de funcionamiento con un tiempo de exposición de dos horas considerando condiciones geográficas y de irradiancia en días soleados, parcialmente nublados y completamente nublados de la ciudad de Ambato se puede obtener temperaturas de calentamiento de agua de $75^{\circ} \mathrm{C}, 50^{\circ} \mathrm{C}$ y $30^{\circ} \mathrm{C}$ respectivamente y en términos de eficiencia en el rango del 30 al 76\% . (Toalombo \& Cabrera, 2011)
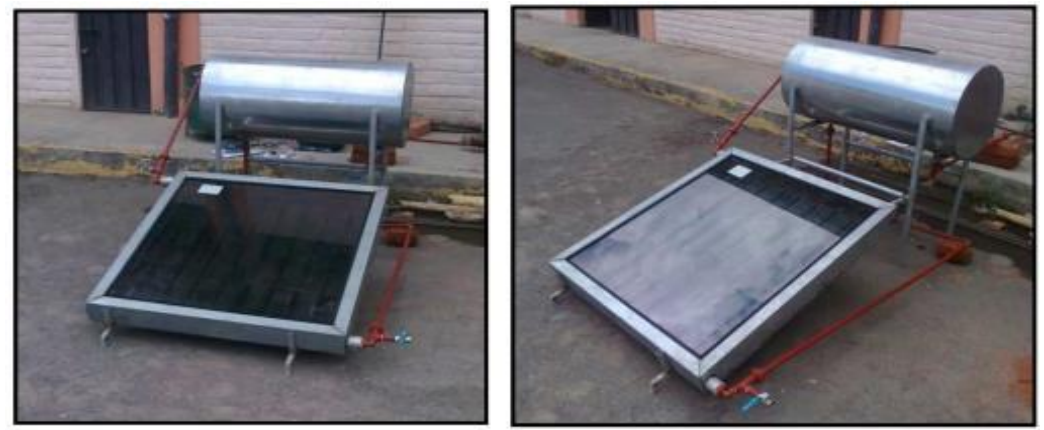

Figura 3. Colector solar

Fuente: Elaboración propia.

- Un calentador de tubos sellados al vacío figura 3, mediante el cual según (Salcedo \& Cabrera, 2011) se puede captar energía de hasta $1854 \mathrm{~W}$, una temperatura máxima de $62^{\circ} \mathrm{C}$ con valores de radiación promedio en un periodo de exposición de seis horas en la ciudad de Ambato.

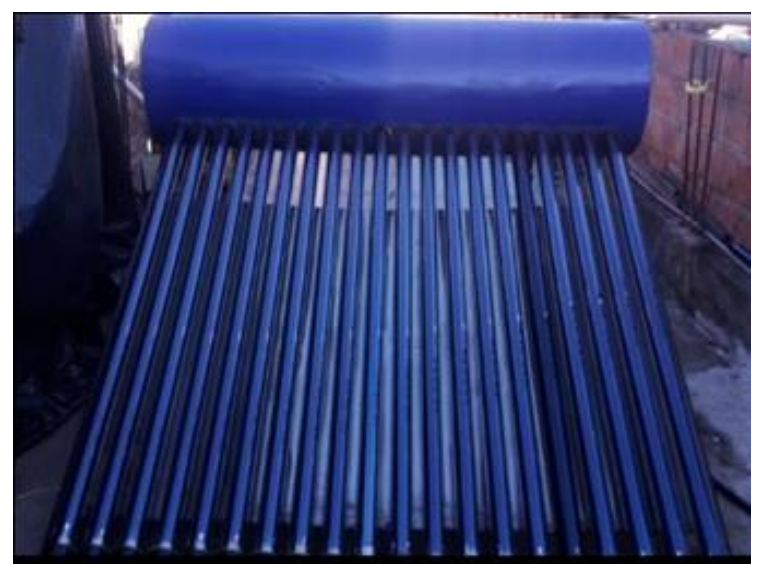

Figura 4. Calentador solar de tubos al vacío

Fuente: Elaboración propia.

El diseño del convertidor se basa en el alcance de lo establecido en la norma NTE INEN 1 912:1992 Calentadores eléctricos de agua para uso doméstico (INEN, 1992). Tomando en cuenta que cuando se tenga condiciones ambientales adversas (temperaturas bajas), o se encuentre trabajando en horas nocturnas o a la madrugada los paneles térmicos no tendrán la capacidad de mantener y/o calentar el agua; por esta razón se opta por añadir al sistema resistencias térmicas tipo sumergidas de Constatan figura 5, su función principal es elevar la temperatura cuando se le suministra corriente eléctrica, para precautelar la seguridad de los usuarios se realiza la conexión debida a tierra mediante una varilla de cobre. 


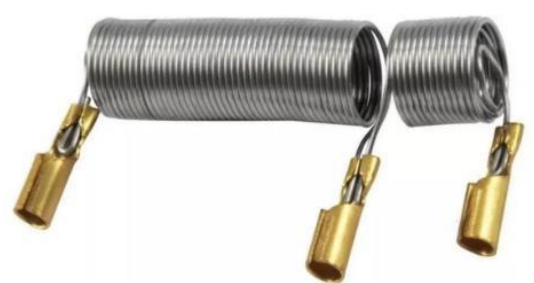

Figura 5. Esquema de resistencias eléctricas

Fuente: (En One, 2019)

En la tabla 1 se muestran alternativas de selección de resistencias que se obtuvieron a través de cálculos.

Tabla 1. Alternativas de diseño de resistencia térmica eléctrica

Fuente: Elaboración propia.

\begin{tabular}{lllll}
\hline & A1 & A2 & A3 & A4 \\
\hline Diámetro (mm) & 1 & 1 & 1 & 0,5 \\
Número espiras $(\mathrm{N})$ & 40 & 20 & 10 & 20 \\
Resistencia $(\boldsymbol{\Omega})$ & 0,941 & 0,470 & 0,235 & 1,882 \\
Intensidad $(\mathrm{A})$ & 12,75 & 25,53 & 51,06 & 6,59 \\
Potencia $(\mathrm{W})$ & 152,97 & 306,36 & 612,76 & 79,08 \\
Duración de las baterías & $15 \mathrm{hrs}$. & $07 \mathrm{hrs}$. & $03 \mathrm{hrs}$. & $30 \mathrm{hrs}$ \\
& $40 \mathrm{~min}$ & $\mathbf{4 9} \mathrm{min}$ & $55 \mathrm{~min}$ & $21 \mathrm{~min}$ \\
\hline
\end{tabular}

Se opta por la alternativa A3 ya que cada resistencia proporciona una potencia superior a los $600 \mathrm{~W}$ y generan calor en el rango comprendido entre 2 a $4 \mathrm{KJ}$ para un total de tres resistencias pese a que se reduce la duración de la batería a una hora, se estima suficiente para el uso en las condiciones adversas.

En el controlador se programa para que active el funcionamiento del convertidor figura 6 , cuando las señales de entrada y salida de los sensores se encuentren en el rango de 25 a $30^{\circ} \mathrm{C}$.



Figura 6. Convertidor

Fuente: Elaboración propia.

También se programa condiciones de carga de batería a saber: 
1. Cuando el nivel de carga de la batería este en un rango de 95 a $100 \%$ el sistema de control desactiva las dos fuentes de energía (solar y eólico) y provoca el frenado en el generador eólico o si la velocidad del viento supera los $20 \mathrm{~m} / \mathrm{s}$.

2. Cuando el nivel de carga de la batería este en un rango de 80 a $94 \%$ el sistema de control activa una fuente siempre y cuando se encuentren generando voltaje superior a los $10 \mathrm{~V}$ o ambas fuentes de energía cuando se encuentren a la mitad de su capacidad hasta alcanzar el rango superior.

3. Cuando el nivel de carga de la batería este en un rango de 71 a $79 \%$ el sistema de control activa las dos fuentes de energía hasta alcanzar el rango superior.

4. Cuando el nivel de carga de la batería este en un rango inferior al $70 \%$ el sistema de control activa la alarma para que los usuarios procedan a desconectar los equipos y activa ambas fuentes hasta alcanzar el rango superior.

\section{Resultados.}

Para validar el funcionamiento del proyecto se ingresan datos al sistema de control provenientes de los paneles fotovoltaicos cada cinco minutos se promedia por hora y por mes mostrados en la figura 7, en un periodo comprendido desde las ocho AM hasta las dieciséis horas PM tiempo en el que la irradiancia supera los $150 \mathrm{~W} / \mathrm{m}^{2}$ y en la cual es apreciable una variación significativa de la temperatura.

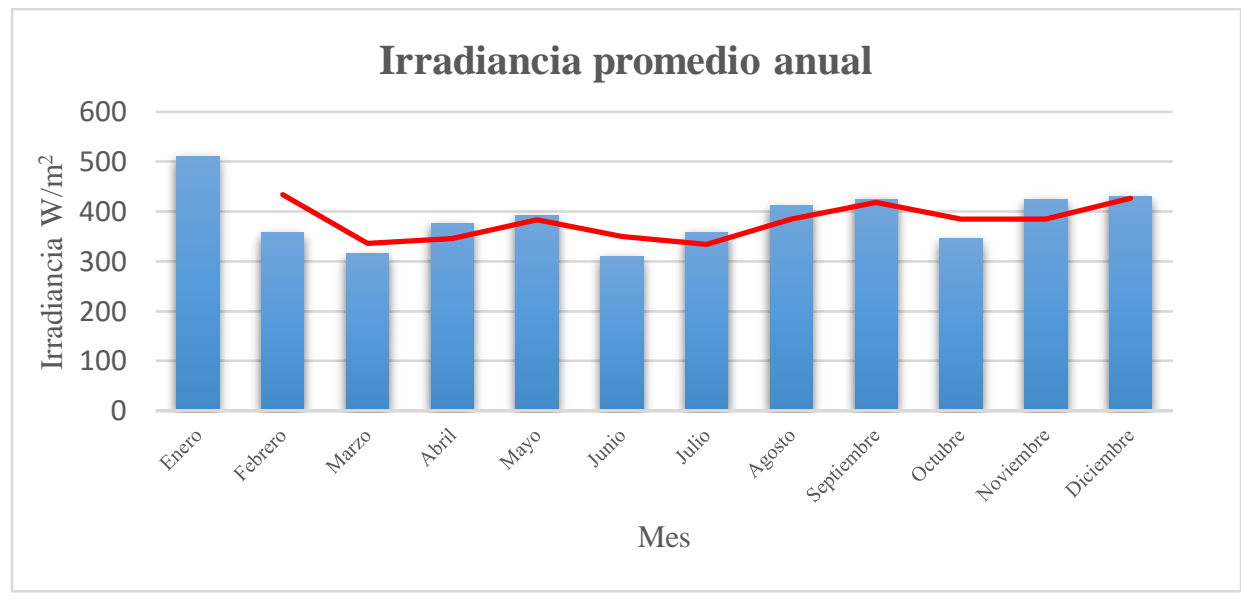

Fiqura 7. Tendencia de irradiancia en la zona

Fuente: Elaboración propia.

Como se puede observar en la figura 7 dependiendo del mes se pueden obtener valores de irradiancia en el rango comprendido de 300 a $500 \mathrm{~W} / \mathrm{m}^{2}$ y en general se tiene un promedio de $350 \mathrm{~W} / \mathrm{m}^{2}$ por tanto se puede aseverar que el lugar cuenta con el suficiente potencial para que el proyecto funcione de manera adecuada.

En cuanto a la temperatura, se parte de la que se encuentra al ambiente en el sector 5 a $7^{0} \mathrm{C}$. Una vez instalados los paneles térmicos se toman datos cuyos resultados se muestran en la figura 8 , se puede apreciar que con el precalentamiento se logra subir la temperatura del agua a un rango de 21 a $23^{\circ} \mathrm{C}$. Para determinar su autonomía se llena el tanque y se permite el consumo hasta que la temperatura sea inferior a los $13^{\circ} \mathrm{C}$, con lo que se estableció en 26 minutos. Posteriormente se permite el funcionamiento del convertidor 
con lo cual se alcanza 8 minutos de autonomía para un total de 34 minutos y el incremento en $4^{0} \mathrm{C}$ provenientes de las resistencias seleccionadas suministrada por la batería instalada y recirculación forzada, logrando de esta manera alcanzar la temperatura de confort establecida en el rango de 25 a $30^{\circ} \mathrm{C}$.

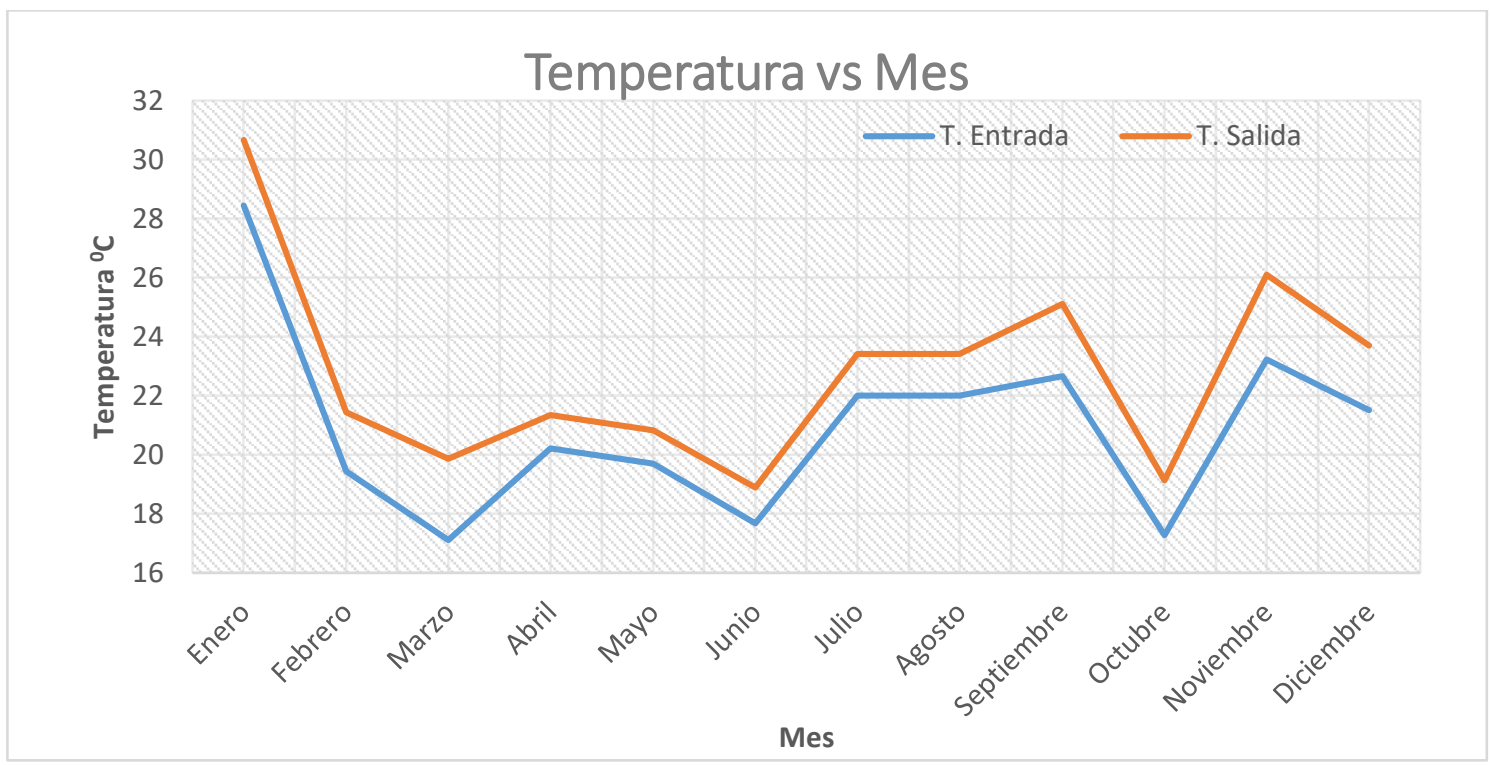

Figura 8. Temperatura vs mes paneles térmicos solares

Fuente: Elaboración propia.

Se determina la eficiencia del convertidor dichos resultados se muestran en la figura 9, de ella se puede concluir que en los meses en que se presente una mayor irradiancia será suficiente la energía generada por los paneles térmicos mientras que en los que la eficiencia se encuentra por debajo del $70 \%$ requerirán de una mayor demanda tanto del convertidor como de las resistencias.

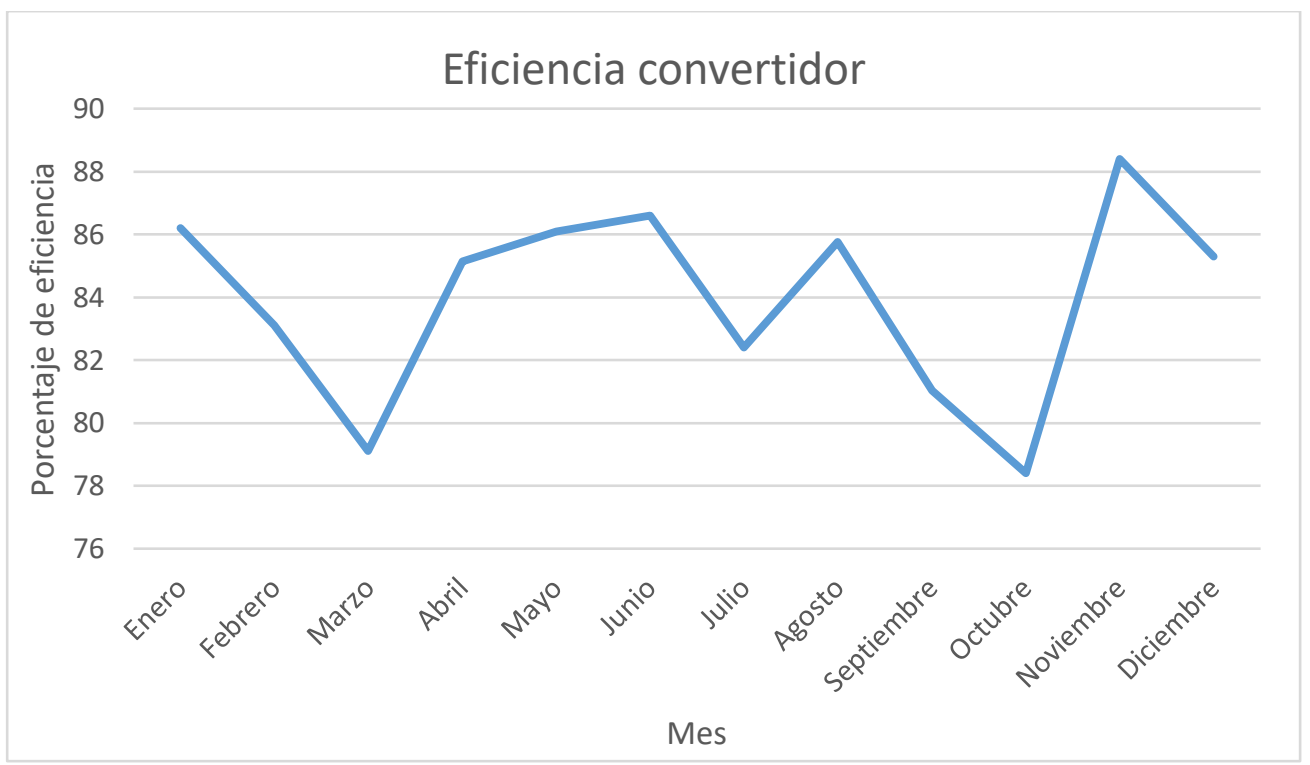

Figura 9. Eficiencia del convertidor por mes Fuente: Elaboración propia.

Según la organización mundial de la salud (OMS, s.f.) se necesita un aproximado de 60 litros de agua caliente en promedio por persona para aseo e higiene personal, se pude 
incrementar a este un $20 \%$ en otros servicios como limpieza, para determinar la autonomía del sistema de calentamiento de agua previamente se determinó el caudal en el sector y con este valor se determina el volumen de agua.

$$
\begin{gathered}
v=Q * t \\
v=\left(1,243 \times 10^{-4} \frac{\mathrm{m}^{3}}{\mathrm{~s}}\right) *(2040 \mathrm{~s}) \\
v=0,2536 \mathrm{~m}^{3} \\
v=253,6 l
\end{gathered}
$$

Se puede concluir que el abastecimiento es suficiente para dotar de agua caliente a cuatro usuarios con un consumo medio bajo.

Para determinar el nivel de carga de las baterías y si el sistema funciona de manera adecuada se provoca las condiciones descritas en el sistema mediante una carga de 200 amperios en un periodo de tiempo desde las ocho AM hasta diecinueve horas PM, la batería se encuentra a plena carga, los datos se muestran en la figura 10, como se puede apreciar la batería comienza a descargarse aproximadamente una hora el sistema detecta que se encuentra por debajo del $70 \%$ y activa las fuentes de energía y la carga de la batería a partir de las doce horas cuando la carga se encuentra al $80 \%$ el sistema activa los paneles solares, frena el generador eólico y carga la batería a partir de las dieciséis horas treinta minutos enciende el generador eólico.

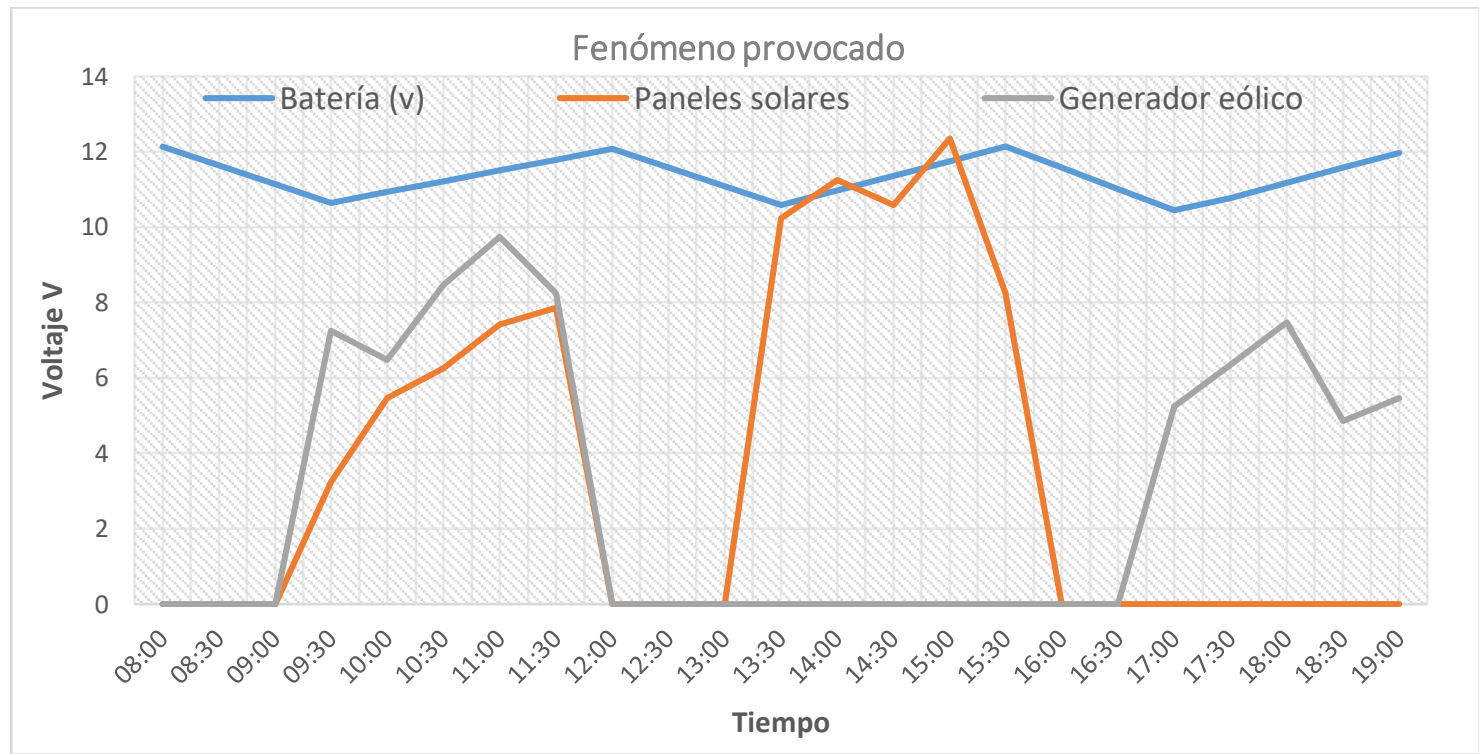

Figura 10. Variación de carga de batería

Fuente: Elaboración propia.

En la figura 11 se muestra como varia el nivel de carga de la batería comparada con el consumo diario durante un periodo de prueba de 80 días. 


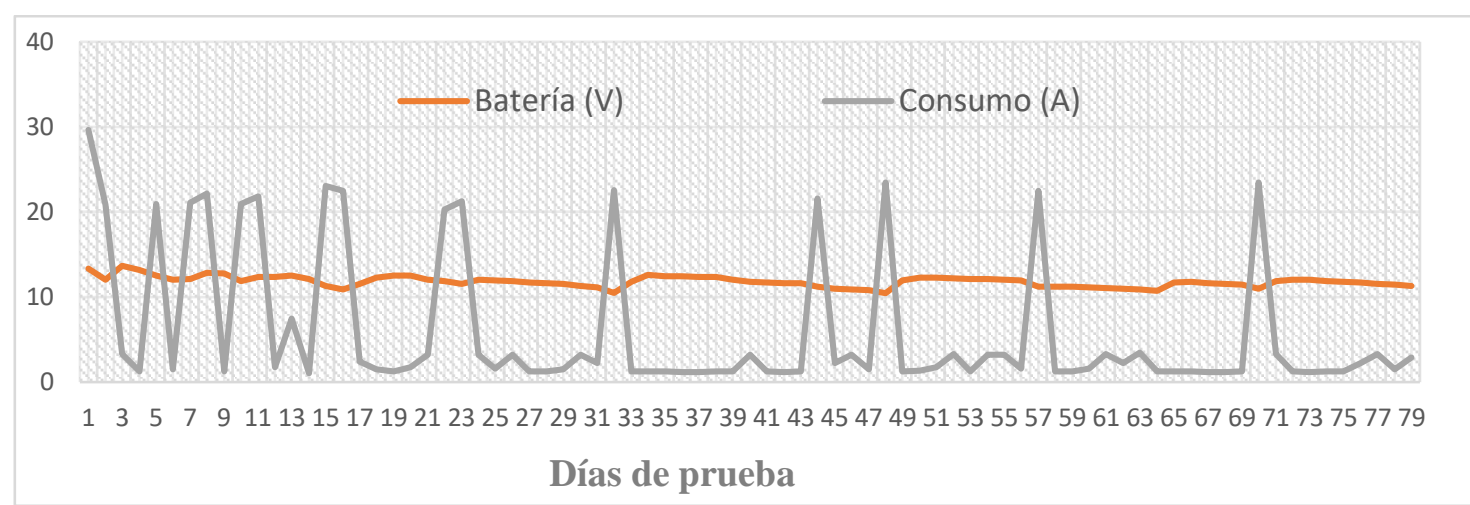

Figura 11. Variación de carga de batería Fuente: Elaboración propia.

En la figura 12 se muestra como el sistema en base al consumo incrementa de 1 a $2^{0} \mathrm{C}$ la temperatura del agua a través del convertidor y las resistencias para encontrase dentro de la temperatura de confort. Estos datos son tomados durante un periodo de tiempo de 80 días.

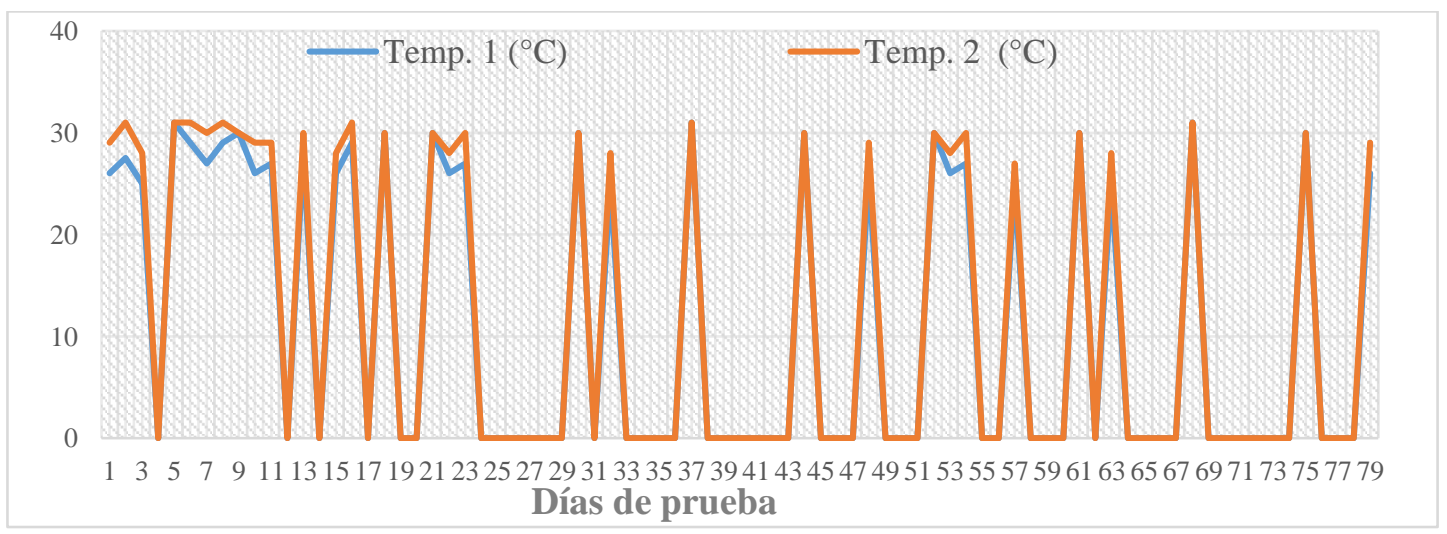

Figura 12. Comportamiento de la temperatura del agua

Fuente: Elaboración propia.

\section{Conclusiones.}

- La temperatura que sumista el convertidor en el rango de 2 a $4^{0} \mathrm{C}$ puede mantenerse hasta por un tiempo de 8 minutos cuando el agua alcanza $12^{0} \mathrm{C}$ en las peores condiciones de funcionamiento del sistema. Esto resulta adecuado si se considera que la temperatura ambiente del lugar es de 5 a $7^{0} \mathrm{C}$.

- En el proyecto se parte de dos módulos solares de $100 \mathrm{~W}$, un aerogenerador de baja potencia de $174 \mathrm{~W}$, dos paneles térmicos solares y dos baterías para el almacenamiento de energía cada una de 6 voltios y $200 \mathrm{Ah}$, en las condiciones actuales los usuarios consumen un total de 67 amperios (se considera también el consumo de la resistencia eléctrica incorporadas al convertidor) y una potencia de $64 \mathrm{~W}$ lo que representa una autonomía de aproximadamente dos horas de suministro con consumo continuo. Si se incrementa el número de baterías al sistema se podrá dotar de mayor capacidad al sistema y mejorar el estilo de vida de los habitantes.

- Para sectores en los cuales no existe suministro de energía eléctrica 
convencional o de acceso limitado al sistema de interconexión nacional proyectos como el descrito son una alternativa viable; en el sector de Yatzaputzán se ha logrado aprovechar recursos no convencionales como el eólico y el solar que han dotado de energía para el uso de equipos básicos que necesitan de ella, y en conjunción con el convertidor, los paneles térmicos solares y las resistencias eléctricas se ha dotado de agua caliente con consumo medio bajo a una temperatura de confort.

\section{Agradecimientos.}

Este proyecto de investigación ha sido financiado por la Dirección de Investigación y Desarrollo (DIDE), de la Universidad Técnica de Ambato en el marco del proyecto: "Medios de conversión de energía eólica - solar a pequeña escala para satisfacer necesidades energéticas básicas en comunidades remotas de la provincia de Tungurahua", aprobado con resolución 1525-CU-P-2018 (Código: PFICM 16), lo cual se agradece.

\section{Referencias bibliográficas.}

Aldaz, A. (2019). Diseño y construcción de un sistema de control para un generador de energía renovable híbrido eólico-fotovoltaico ubicado en el sector de Río Blanco comunidad de Yatzaputzán provincia de Tungurahua. Universidad Técnica de Ambato.

Carrillo, M., Romero, J., \& Mayorga, A. (2020). Evaluation of the Performance of a Low Power Wind Turbine Applied to Isolated Communities of the Andean Region in Ecuador. (Springer, Ed.) International Conference on Innovation and Research, 515-530.

Colmenar, A., Borge, D., Collado, E., \& Castro, E. (2015). Generación distribuida, autoconsumo y redes inteligentes . Madrid, España: UNED.

En One. (15 de Agosto de 2019). Obtenido de http://enone.pe/producto/resistenciaoriginal-para-ducha-3t-5500w-lorenzetti-1-pieza/

Espín, F. (2019). Diseño e implementación de un software para evaluar el comportamiento en tiempo real de un sistema híbrido eólico-solar en el sector de Río Blanco comunidad Yatzaputzán. Ambato: Universidad Técnica de Ambato.

Ethernet library. (s.f.). Ethernet Shield Módule. Recuperado el 20 de Julio de 2019, de https://www.arduino.cc/en/Reference/Ethernet

Geek Factory. (s.f.). Tarjeta genérica compatible con Arduino Uno SMD CH340. $\begin{array}{llllll}\text { Recuperado el } 20 \text { de Julio de } & \end{array}$ https://www.geekfactory.mx/tienda/sensores/sensor-voltaje-ac-zmp101b/ 
Guananga, M. A. (2017). Diseño y construcción de un sistema fotovoltáico de baja potencia en el sector Rio Blanco perteneciente a la comunidad de Yatzaputzan.

INEN. (1992). Calentadores eléctricos de agua para uso doméstico. Requisitos. Instituto Ecuatoriano de Normalización, Quito.

Méndez, J. M., \& García, R. C. (2007). Energía solar fotovoltaica (Segunda ed., Vol. I). Madrid: FC Editorial.

Moreta, L. (2019). Diseño de un convertidor que permita transformar la energía eléctrica obtenida mediante un sistema de generación híbrido compuesto por paneles solares fotovoltaicos y un generador eólico en energía térmica. Universidad Técnica de Ambato.

Naranjo, J. (2017). Determinación del potencial eólicodisponible en el sector de Río Blanco perteneciente al Cantón Ambato provincia de Tungurahua. Universidad Técnica de Ambato.

Ogata, K. (2010). Ingeniería de control moderna. Madrid: Prentice Hall.

OMS. (s.f.). Organización Mundial de la salud. Recuperado el 25 de Noviembre de 2019, de https://www.who.int/globalchange/ecosystems/water/es/

Prats, D., García, R., \& Alonso, J. (Abril de 2011). Sistemas híbridos con base en las energías renovables para el suministro de energía a plantas desaladoras. Ingeniería Mecánica, XIV(1), 22-30.

Salcedo, V., \& Cabrera, S. (2011). Influencia del estudio de la tecnología de vacío en tubos sobre la eficiencia para el calentamiento de agua usando la energia solar. Universidad Técnica de Ambato.

Toalombo, B., \& Cabrera, S. (2011). Estudio del espectro de irradiación solar para determinar el potencial de energía aprovechable en la ciudad de Ambato. Universidad Técnica de Ambato,

\section{【Ciencia}




\section{PARA CITAR EL ARTÍCULO INDEXADO.}

Carrillo Rosero, C. M., Aldaz Toaza, A. A., Moreta López, L. D., \& Mayorga Pardo, A. S. (2021). Diseño y control de un convertidor de energía eléctrica a térmica, usando como fuente un sistema híbrido eólico-solar. ConcienciaDigital, 4(2), 185-197. https://doi.org/10.33262/concienciadigital.v4i2.1657

\section{LCiencia}

El artículo que se publica es de exclusiva responsabilidad de los autores y no necesariamente reflejan el pensamiento de la Revista Conciencia Digital.

El artículo queda en propiedad de la revista y, por tanto, su publicación parcial y/o total en otro medio tiene que ser autorizado por el director de la Revista Conciencia Digital.

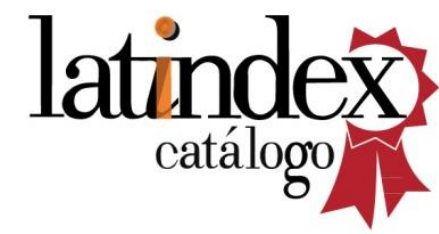

